\title{
Review Article: The Effect of Performing a Paracentesis on Hospital Outcomes
}

\author{
Lindsay A Sobotka*, Khalid Mumtaz ${ }^{*}$ \\ Department of Gastroenterology, Hepatology and Nutrition, The Ohio State Wexner Medical Center, USA
}

Article Info

\section{Article Notes}

Received: September 11, 2018

Accepted: October 18, 2018

\section{${ }^{*}$ Correspondence:}

${ }^{*}$ Dr. Lindsay A Sobotka, Department of Gastroenterology, Hepatology and Nutrition, The Ohio State Wexner Medical Center, USA; E-mail: Lindsay.Sobotka@osumc.edu. "Dr. Khalid Mumtaz, Department of Gastroenterology, Hepatology and Nutrition, The Ohio State Wexner Medical Center, USA; E-mail: khalid.mumtaz@osumc.edu.

(C) 2018 Sobotka LA, Mumtaz K. This article is distributed under the terms of the Creative Commons Attribution 4.0 International License.

\section{Keywords:}

Paracentesis

Ascites

Hospital outcomes
Abstract

Decompensated cirrhosis with ascites results in high health care expenditures, 30 day readmission, morbidity, and mortality. Paracentesis is indicated in patients with cirrhosis and ascites to rule out spontaneous bacterial peritonitis and for symptomatic control. Performing at least a diagnostic paracentesis has been proved to reduce inpatient mortality; however, the procedure was also associated with longer length of stay, higher costs during hospitalization and increased risk of 30-day readmission. In summary, diagnostic paracentesis is crucial to rule out infection, but other interventions should be utilized to control ascites, as worse hospital outcomes as likely associated with a large volume paracentesis.

The prevalence of cirrhosis is gradually increasing, not only in the United States but also across the world ${ }^{1}$. Cirrhosis is associated with high healthcare expenditures, morbidity, and mortality especially once these patients develop decompensated disease ${ }^{2,3}$.

One of the most prevalent and early features of decompensation is the development of ascites ${ }^{4}$. The accumulation of ascites with associated discomfort and shortness of breath is one of the top ten reasons for readmission in patients with decompensated cirrhosis 5 . Multiple treatment options exist in the management cirrhosis including fluid and salt restriction, diuretics, large volume paracentesis, transjugular intrahepatic portosystemic shunt (TIPS) and liver transplantation. Recent literature has suggested that some of these interventions may have negative effects on hospital outcomes ${ }^{5-8}$.

Paracentesis is a low risk, well tolerated procedure that is utilized in patients with decompensated cirrhosis with ascites ${ }^{9}$. Paracentesis is helpful in diagnosing spontaneous bacterial peritonitis (SBP) or to relieve symptom when patients develop tense, symptomatic ascites. Unfortunately, paracentesis is only a temporizing measure in patients who develop recurrent abdominal ascites ${ }^{10}$.

Given paracentesis is commonly utilized for management of ascites, it is important review the literature in order to analyze the effect this procedure has on hospital outcomes. In a time where health care utilization and cost are at an all-time high, it is crucial to perform interventions that not only improve morbidity and mortality but improve hospital outcomes and minimize cost. In addition, reimbursement for hospitalization will soon be based on the physicians and hospital's ability to perform cost conscious care ${ }^{11}$.

According to a nationwide study performed by Bajaj and associates, patients with decompensated cirrhosis have a $53 \%$ 
chance of 90-day readmission. This study determined that patients with a higher Model for Endstage Liver Disease (MELD) score, diabetes, prior hepatic encephalopathy, and those taking prophylactic antibiotics were more likely to be readmitted ${ }^{6}$. An additional nationwide study conducted by Scaglione also revealed a $50 \%$ early readmission rate in patient with decompensated cirrhosis and determined readmission to be associated with blood transfusion, TIPS, paracentesis, thoracentesis, and upper endoscopy ${ }^{7}$. Our study utilizing the Nationwide Inpatient Sample specifically analyzed readmission rates in patient with decompensated cirrhosis with ascites and determined this specific group of patients had a $30 \%$ chance of 30 day readmission. Readmission in these patients was associated with younger age, government funded insurance, multiple comorbidities, nonalcoholic cirrhosis and hepatocellular carcinoma ${ }^{5}$. Another study by Volk et al predict that about $22 \%$ of these readmissions could have been prevented by better patient education and intensive outpatient monitoring 8 .

Not only patients with decompensated cirrhosis have higher rates of unplanned 30-day readmission, but once these patients are readmitted, they had higher rate of 90 days, 1 year and overall mortality compared to patients that were not readmitted in addition to higher health care costs ${ }^{7}$.

Other studies focused on evaluating the effect on patients and hospital outcomes of certain interventions for patient with decompensated cirrhosis, specifically the utilization of paracentesis in patients with ascites. A study utilizing the Nationwide Inpatient Sample performed by Orman et al determined that patients with clinical indications for paracentesis only underwent a paracentesis approximately $60 \%$ of the time. If these patients underwent paracentesis within 1 day of admission, they had improved short term outcomes, specifically mortality, due to the prompt recognition of SBP or improved symptomatic control ${ }^{12}$. While the utilization of paracentesis for the diagnosis of SBP is associated with improved mortality, other hospital outcomes were worse. Length of stay for patients that underwent paracentesis was longer according to Orman and hospital costs were significantly higher according to studies performed by both Orman and Sobotka ${ }^{5,12}$. In addition, patients that underwent paracentesis were more likely to be readmitted within 30 days $^{5}$. It is likely that the patients in these studies underwent a large volume paracentesis for symptom management in addition to diagnostic paracentesis which would have increased cost associated with procedure and is only a temporary measure for control of ascites, therefore patients would be more likely to be readmitted when ascites reaccumulated. However, given limitation in International Classification of Diseases (ICD) 9 codes used in these studies, reviewers are only able to determine that a patient underwent a paracentesis, not specifically a diagnostic, therapeutic or both.

While the majority of the reported studies have some limitations, specifically with the inability to determine between a large volume paracentesis and diagnostic paracentesis, some conclusions can be made. Patients with decompensated cirrhosis are at high risk of inpatient mortality, longer length of stay, and 30 day readmission. These patients have about a $30 \%$ to $50 \%$ chance of unplanned, early readmission. Patients that are readmitted have higher rates of 90 days, 1 year, and overall mortality compared to patients that are not readmitted. If these patients have ascites and undergo a paracentesis for treatment, they have improved short term outcomes, likely due to prompt diagnosis of SBP and timely evacuation of ascites to assist with symptom control. However, mostly they undergo a simultaneous therapeutic paracentesis for management of symptomatic ascites and therefore, have increased length of stay, hospital costs and rates of 30 day readmission. Alternative methods for long term management of recurrent ascites such as early TIPS, drainage pumps, and exception points to expedite liver transplantation may improve the healthcare utilization in these patients.

\section{References}

1. Wong MCS, Huang J. The Growing Burden of Liver Cirrhosis: Implications for Preventative Measures. Hepatol Int. 2018; 12: 201. DOI: $10.1007 / \mathrm{s} 12072-018-9865-\mathrm{y}$

2. Neff GW, Duncan CW, Schiff ER. The Current Economic Burden of Cirrhosis. Gastroenterology \& Hepatology. 2011; 7(10): 661-671.

3. Samonakis DN, Koulentaki M, Coucoutsi C, et al. Clinical outcomes of compensated and decompensated cirrhosis: A long term study. World J Hepatol. 2014; 6: 504-512.

4. Nusrat S, Khan MS, Fazili J, et al. Cirrhosis and its complications: evidence based treatment. World J Gastroenterol. 2014; 20: 5442-5460.

5. Sobotka LA, Modi RM, Vijayaraman A, et al. Paracentesis in cirrhotics is associated with increased risk of 30-day readmission. World Journal of Hepatology. 2018; 10(6): 425-432. doi:10.4254/wjh.v10.i6.425.

6. Bajaj JS, Reddy KR, Tandon P, et al. The Three-Month Readmission Rate Remains Unacceptably High in a Large North American Cohort of Cirrhotic Patients. Hepatology (Baltimore, Md). 2016; 64(1): 200 208. doi:10.1002/hep.28414

7. Scaglione SJ, Metcalfe L, Kliethermes S, et al. Early Hospital Readmissions and Mortality in Patients With Decompensated Cirrhosis Enrolled in a Large National Health Insurance Administrative Database. J Clin Gastroenterol. 2017; 51: 839-844.

8. Volk ML, Tocco RS, Bazick J, et al. Hospital Re-Admissions among Patients with Decompensated Cirrhosis. The American journal of gastroenterology. 2012; 107(2): 247-252. doi:10.1038/ajg.2011.314.

9. De Gottard A, Thevenot T, Spahr L, et al. Risk of Complications After Abdominal Paracentesis in Cirrhosis Patients: A Prospective Study. Clinical Gastroenterology and Hepatology. 2009; 7: 906-909. DOI: 10.1016/j.cgh.2009.05.004

10. Sudulagunta SR, Sodalagunta MB, Bangalore Raja SK, et al. Clinical Profile and Complications of Paracentesis in Refractory Ascites Patients With Cirrhosis. Gastroenterology Res. 2015; 8: 228-233. 
11. Readmissions Reduction Program. Available from: https: // www.cms.gov/medicare/medicare-fee-for-service-payment/ acuteinpatientpps/readmissions-reduction-program.html
12. Orman ES, Hayashi PH, Bataller R, et al. Paracentesis is associated with reduced mortality in patients hospitalized with cirrhosis and ascites. Clin Gastroenterol Hepatol. 2014;1 2: 496-503. 